OPEN ACCESS

Edited by:

Paula Soares,

University of Porto, Portugal

Reviewed by:

José Manuel Lopes,

University of Porto, Portugal

Erdogan Pekcan Erkan,

University of Helsinki, Finland

*Correspondence:

Reety Arora

reetya@ncbs.res.in

†These authors have contributed equally to this work and share first authorship

${ }^{\ddagger}$ Present address: Komal Gupta,

Medical Research Council Cancer Unit, University of Cambridge,

Cambridge, United Kingdom Anjali Vijaykumar Imperial College London, London, United Kingdom

Specialty section:

This article was submitted to

Molecular Diagnostics and

Therapeutics,

a section of the journal

Frontiers in Molecular Biosciences

Received: 18 October 2019

Accepted: 14 January 2020

Published: 04 February 2020

Citation:

Arora R, Gupta K, Vijaykumar A and Krishna S (2020) DETECTing Merkel Cell Polyomavirus in Merkel Tumors.

Front. Mol. Biosci. 7:10

doi: 10.3389/fmolb.2020.00010

\section{DETECTing Merkel Cell Polyomavirus in Merkel Tumors}

\author{
Reety Arora ${ }^{1 * \neq}$, Komal Gupta $^{1,2+\neq}$, Anjali Vijaykumar ${ }^{1 \neq}$ and Sudhir Krishna ${ }^{1}$ \\ ${ }^{1}$ National Centre for Biological Sciences, Tata Institute of Fundamental Research (TIFR), Bangalore, India, ${ }^{2}$ Department of \\ Biology, Indian Institute of Science Education and Research, Pune, India
}

Merkel cell carcinoma (MCC) is a rare, aggressive skin cancer caused either by Merkel cell polyomavirus (MCV) T antigen expression, post-integration ( $80 \%$ cases), or by UV-mediated DNA damage. Interestingly, overall survival of MCV-positive Merkel cell carcinoma patients is better, making this differential information of significant diagnostic and prognostic value. Also, MCV provides a direct target for therapy in MCC patients. Currently, the methods used for diagnosis of MCV in tumors are often discordant and unreliable. Here we used a guided molecular scissors based-DNA Endonuclease Targeted CRISPR Trans Reporter (DETECTR) technique to develop an in vitro molecular diagnostic tool for MCV-positive MCC. DETECTR couples recombinase polymerase based amplification of target MCV DNA with Cas12a mediated detection. CRISPR diagnostics couple specific detection followed by cutting of the pathogenic DNA by the Cas enzyme-gRNA complex, with non-specific cutting of ssDNA that provides a measurable visual cue. To detect MCV DNA in MCC, we designed Cas12a gRNAs targeting the MCV DNA and tested their targeting efficiency, and sensitivity using a fluorophore quencher labeled reporter assay. We show that MCV DETECTR system can detect MCV integrated in Merkel tumor rapidly, specifically and with femto-molar sensitivity. Our study is a preliminary, proof-of-principle analysis showing the use of CRISPR for MCV diagnosis. Further validation in human tumor samples is needed for its clinical use in the near future. This new system is promising and we hope it can be coupled with immunohistochemical studies to diagnose the viral status of MCC in clinics soon.

Keywords: Merkel cell carcinoma, Merkel cell polyomavirus (MCPyV), DETECTR, Merkel tumors, molecular diagnosis, CRISPR, CRISPR diagnosis, Cas12a

\section{INTRODUCTION}

Merkel Cell Carcinoma (MCC) is a rare and aggressive neuroendocrine skin cancer (Becker et al., 2017). MCC is associated with old age, excessive UV exposure and weak immune system (Engels et al., 2002; Harms et al., 2018). It is either caused by Merkel cell polyomavirus (MCV) (Feng et al., 2008) or long term Ultraviolet (UV) exposure (Harms et al., 2015; Goh et al., 2016; Starrett et al., 2017).

$\mathrm{MCV}$ is the latest addition to the group of human oncoviruses and the only known human oncovirus in the polyomavirus family (Feng et al., 2008). The virus has a double stranded DNA genome, encoding for early and late region genes. Early region genes encode for Small and Large Tumor antigens (sT and LT, respectively) and late region genes encode for the viral proteins (VP) (Feng et al., 2008; Harms et al., 2018). 
In virus positive cancers MCV-dependent carcinogenesis requires two events. First, the viral genome gets integrated into the host cell genome, followed by LT getting truncation mutations in its DNA binding and helicase domains to make the virus replication incompetent (Shuda et al., 2008). The expressed $\mathrm{T}$ antigens then lead to oncogenesis by altering several pathways and are required for proliferation and survival of MCC cells (Houben et al., 2010, 2012; Shuda et al., 2014). A majority of MCC cases have been found to be MCV positive ( $80 \%)$ (Feng et al., 2008; Shuda et al., 2009; Becker et al., 2017; Harms et al., 2018).

Both viral positive and viral negative Merkel tumors show identical phenotypes, immunohistochemical (IHC) staining patterns and occur at similar sun-exposed locations on the human body (Hodgson, 2005; Arora et al., 2012, 2019; DeCaprio, 2017; Moore and Chang, 2017; Moshiri et al., 2017; Harms et al., 2018). A pathologist is unable to distinguish between the two types of tumors without the aid of Next-Gen-Sequencing (Goh et al., 2016; Harms et al., 2016). Studies determining the prognostic role of $\mathrm{MCV}$ status have had contradictory results, showing either no difference or worsened prognosis of virus negative MCC (Schrama et al., 2011; Hall et al., 2012; Moshiri et al., 2017; Harms et al., 2018). In a recent and the largest study thus far, Moshiri et al. showed that out of 282 MCC tumors, 20\% were MCV negative using multimodal qPCR and immunohistochemistry analysis (Moshiri et al., 2017). Interestingly, the study indicated that although both often have aggressive and fatal disease courses, as compared to MCV negative MCC patients, MCV positive MCC patients had significantly better progression-free survival, MCC-specific survival, and overall survival from MCC. Hence identifying the viral status is not only diagnostically (as the cancer cause) important, but important for prognostic predictability as well.

Besides diagnosis and prognosis, the knowledge of $\mathrm{MCV}$ integration and $\mathrm{T}$ antigen expression in MCC serves are a promising piece of information for MCC treatment as well. The viral DNA sequences and proteins serve as significant external targets that can be exploited for directed therapy for MCV positive cases.

Currently, most commonly used techniques for detecting MCV in MCC tumors include amplifying MCV DNA from DNA isolated from MCC tumors using PCR or immunohistochemistry (IHC) staining for MCV LT using monoclonal antibodies (Guastafierro et al., 2013; Becker et al., 2017; DeCaprio, 2017; Moshiri et al., 2017; Harms et al., 2018). However, these techniques are not the most concordant and reliable. For instance, the Moshiri study using 282 MCC samples used three techniques, IHC with CM2B4 and Ab3 and PCR, for identifying the viral status (Moshiri et al., 2017). However, only 167 / 282 cases showed same viral status in all the three techniques whereas of 282 total cases, 199 cases were positive by qPCR, 205 by CM2B4-IHC, and 254 by Ab3-IHC. Besides, these techniques are usually tedious and required trained personnel to perform them. Thus, there is a need for developing an accurate, sensitive and quick system for distinguishing viral negative vs. viral positive MCC cases (Bhatia et al., 2010; Leroux-Kozal et al., 2015; Brummer et al., 2016; Eid et al., 2017; Harms et al., 2018). In this study we developed a CRISPR/Cas12a based in vitro molecular diagnostic system for detecting MCV.

CRISPR (Clustered Regularly Interspaced Short Palindromic Repeats)/Cas system is a genome editing technology derived from the bacterial immune system (Barrangou et al., 2007). CRISPR uses a guide RNA (gRNA) molecule that targets a Cas endonuclease to a specific genomic site using sequence homology and PAM (Protospacer adjacent motif) recognition. Upon target binding Cas protein induces a double strand break in the target (Hsu et al., 2014; Swarts et al., 2017; Gupta et al., 2019). Cas12a (Also known as Cpf1) is a type V CRISPR protein having various properties distinct from Cas9. Cas12a enzymes recognize a T nucleotide-rich protospacer-adjacent motif (PAM), catalyze their own guide CRISPR RNA (crRNA) maturation, and generate a PAM-distal dsDNA break with staggered 5' and 3' ends (Barrangou et al., 2007; Dong et al., 2016; Murugan et al., 2017). Interestingly, unlike Cas9, after dsDNA target binding the Cas12a enzyme leads to indiscriminate trans ssDNA cleavage activity (Chen et al., 2018). Chen et al. used this property to develop DNA endonuclease-targeted CRISPR trans reporter (DETECTR) system which can rapidly and specifically detect target HPV DNA (Chen et al., 2018).

We adapted the DETECTR system to detect the presence of MCV DNA integration in the Merkel cell tumor genome. Briefly, we assemble a reaction mix containing AsCas12a, MCV specific gRNA, test DNA, and fluorophore-quencher (FQ) ssDNA substrate in a tube. In the presence of MCV DNA, Cas12a binds and cleaves the cis target DNA followed by trans cleavage of fluorescently labeled ssDNA. This Cas12a mediated DNase activity leads to fluorescence based detection of MCV.

Here we show that when combined with Recombinase Polymerase based amplification of target DNA, DETECTR system can identify MCV with femtomolar sensitivity. DETECTR can detect $\mathrm{MCV}$ in MCV positive MCC cells efficiently and specifically. We tested our system on MCC cell lines as a preliminary validation of the system. Testing of this assay on clinical human MCC samples is still required and will add further credibility to our study. Thus, we hope that this MCV DNA detecting system can then be coupled with histopathological and immunohistochemical studies to diagnose the viral status in MCC and help guide clinicians in the near future.

\section{MATERIALS AND METHODS sgRNA Design}

Ten AsCpf1 gRNAs were designed to target the Non-coding Control Region (NCCR), small and large Tumor (sT and LT, respectively) antigen of MCV. Two CRISPR gRNA design tools: Benchling (https://benchling.com/pub/cpf1) and RGEN (www.rgenome.net/cas-designer) were used. MKL-1 (Genbank Accession \#: FJ173815.1) and MS-1 (Genbank Accession \#: JX045709.1) MCV sequences were used as target DNA sequences. gRNAs with highest specificity score and with lowest possible off targets were selected. MCV gRNA target region sequence conservation was analyzed using NCBI BLAST (https://blast. ncbi.nlm.nih.gov/Blast.cgi). 


\section{Synthesis of sgRNAs Using IVT}

For in vitro transcription (IVT), two oligonucleotides were designed as DNA templates, per gRNA. Forward oligonucleotide for AsCas12a gRNA synthesis consisted of the T7 promoter (TAATACGACTCACTATAGG) followed by AsCas12a sgRNA scaffold and 20-nucleotide long guide RNA. Three G's were added after T7 promoter sequence for efficient T7 transcription.

Target substrate for AsCas12a NCCR gRNA1 in vitro cleavage (pam/TARGET):

5' GGCCTCTCTCTTTTtttc CAGAGGCCTCGGAGGCT AGGAGCCCCAAGCCTCTG 3'

crRNA oligo for in vitro transcription (T7 ADDED G/SCAFFOLD/GUIDE):

Forward oligo:

TAATACGACTCACTATAGGGTAATTTCTACTCT

TGTAGATCAGAGGCCTCGGAGGCTAGG

Reverse oligo:

CCTAGCCTCCGAGGCCTCTGATCTAC

AAGAGTAGAAATTACCCTATAGTGAGTCGTATTA

Forward and reverse oligonucleotides were annealed by heating to $95^{\circ} \mathrm{C}$ for $5 \mathrm{~min}$ and slow cooling on bench top. sgRNAs were synthesized using the MEGAShortScript ${ }^{\mathrm{TM}}$ Kit (\# AM1354, Invitrogen) following the manufacturer's protocol with $2 \mu \mathrm{g}$ annealed oligonucleotides template, overnight at $37^{\circ} \mathrm{C}$. The RNA was treated with $1 \mu \mathrm{l}$ of DNase TURBO for $15 \mathrm{~min}$ followed by purification using the MEGAclear Transcription Clean-Up Kit (\# AM1908, Invitrogen) (see Supplementary Table 1 for all oligonucleotides and primer details).

\section{In vitro Cleavage Assay}

In vitro cleavage reaction was performed at $37^{\circ} \mathrm{C}$ in cleavage buffer consisting of $20 \mathrm{mM}$ HEPES ( $\mathrm{pH} \mathrm{7.5),} 150 \mathrm{mM} \mathrm{KCl}$, $10 \mathrm{mM} \mathrm{MgCl}_{2}, 1 \%$ glycerol and $0.5 \mathrm{mM}$ DTT. Thirty nanometers AsCas12a (\#1081068, IDT) and 36 nM gRNA were pre-assembled in the cleavage buffer at $37^{\circ} \mathrm{C}$ for $10 \mathrm{~min} .18 .5 \mathrm{nM}$ dsDNA target was added to the reaction $(20 \mu \mathrm{l})$. PCR amplicons of NCCR, sT, and LT regions from plasmid RAZ2 (Addgene \#114382) were used as dsDNA target templates. The PCR amplicon was purified using Ampure beads (\#A63881, Beckman Coulter) as per manufacturer's protocol. For M13 cleavage assays, $30 \mathrm{nM}$ AsCas 12 and $36 \mathrm{nM}$ gRNA were preassembled at $37^{\circ} \mathrm{C}$ for $10 \mathrm{~min}$ in cleavage buffer. Forty nanometers dsDNA activator and $10 \mathrm{nM}$ single stranded M13mp18 phage (\#N4040S, NEB) were added to initiate the reaction $(30 \mu \mathrm{l})$. The reaction was incubated at $37^{\circ} \mathrm{C}$ for $60 \mathrm{~min}$. The IVC reactions were stopped by treatment with $2 \mu \mathrm{l}$ of Proteinase $\mathrm{K}(10 \mathrm{mg} / \mathrm{ml})$ at $55^{\circ} \mathrm{C}$ for $10 \mathrm{~min} .1 \times$ gel loading dye was added to the reactions and samples were run on $2 \%$ agarose gels (\# RM273, HIMEDIA).

\section{Fluorophore Quencher (FQ)-Labeled Reporter Assays}

$200 \mathrm{nM}$ AsCas12a, $250 \mathrm{nM}$ gRNA and $18.5 \mathrm{nM}$ dsDNA target template were pre-assembled in a $5 \mu \mathrm{l}$ reaction for $30 \mathrm{~min}$ at $37^{\circ} \mathrm{C}$. Reaction was initiated by diluting these complexes to AsCas12a: gRNA: dsDNA template to $50 \mathrm{nM}: 62.5 \mathrm{nM}: 4.6 \mathrm{nM}$ in $1 \mathrm{x}$ Binding buffer $(20 \mathrm{mM}$ Tris- $\mathrm{HCl}, \mathrm{pH} 7.5,100 \mathrm{mM} \mathrm{KCl}$, $5 \mathrm{mM} \mathrm{MgCl} 2,1 \mathrm{mM}$ DTT, 5\% glycerol, $50 \mu \mathrm{g} \mathrm{ml}^{-1}$ heparin) and $50 \mathrm{nM}$ custom ssDNA FQ reporter substrate (IDT). The custom ssDNA reporter substrate consisted of $5^{\prime}$ 6-FAM ${ }^{\mathrm{TM}}$, 5 nucleotides long oligo (TTATT) and $3^{\prime}$ Iowa Black ${ }^{\circledR}$ FQ. The $20 \mu \mathrm{l}$ reactions were incubated at $37^{\circ} \mathrm{C}$ for $1 \mathrm{~h}$ in a 384 well-microplate format. The fluorescence was measured using Tecan (Infinite M Plex) (Excitation: 485 nm, Emission: $535 \mathrm{~nm}$ ). Experiments were repeated independently three times with two technical repeats each time. The graphs were drawn using GraphPad Prism and statistical significance was calculated using one way ANOVA.

\section{Cell Culture}

HEK293T, U20S, and BJhTERT cell lines were obtained from ATCC and grown in Dulbecco's Modified Eagle Medium (DMEM) supplemented with 10\% Fetal Bovine Serum (FBS), 1X Penicillin/Streptomycin (Pen Strep, catalog \# 15140122). Merkel Cell Carcinoma cell line MKL-1 was obtained from ECACC (\# 09111801) and MS-1, MCC26, and WaGa cells were kind gifts from Dr. James Decaprio's laboratory at Dana Farber Cancer Institute, Boston. MCC cell lines were cultured in Roswell Park Memorial Institute (RPMI) Media with 10\% FBS and $1 \mathrm{X}$ Penicillin/Streptomycin. The cells were grown at $37^{\circ} \mathrm{C}$ with $5 \% \mathrm{CO}_{2}$.

\section{Genomic DNA Isolation}

$10^{6}$ cells were harvested for genomic DNA extraction. Cells were lysed in Tris EDTA buffer and $200 \mu \mathrm{g} / \mathrm{mL}$ Proteinase $\mathrm{K}$ at $55^{\circ} \mathrm{C}$ for $1 \mathrm{~h}$. After Proteinase $\mathrm{K}$ was heat inactivated, $1 \mu \mathrm{l}$ of this lysed sample was used for DETECTR experiments.

\section{Recombinase Polymerase Reaction (RPA)}

For DETECTR experiments, genomic DNA was first amplified using recombinase polymerase reaction (RPA). The RPA reaction mix was prepared by adding $0.48 \mu \mathrm{M}$ forward primer and reverse primers, $29.5 \mu \mathrm{l}$ primer Free Rehydration buffer, $1 \mu \mathrm{l}$ of extracted genomic DNA and water to $13.2 \mu$ l (Total volume $47.5 \mu \mathrm{l})$. The reaction mix was added to one TwistAmp ${ }^{\circledR}$ Basic (\#TABAS03KIT, TwistDx) reaction tube. $2.5 \mu \mathrm{l}$ of $280 \mathrm{mM}$ Magnesium Acetate (MgOAc) was added and mixed to start the reaction. The reaction was incubated at $37^{\circ} \mathrm{C}$ for $20 \mathrm{~min}$.

\section{DETECTR Assay for Genomic DNA}

DETECTR uses RPA reaction to amplify target DNA followed by Cas12a based detection. Eighteen microliter of the RPA reaction mix was assembled with $2 \mu \mathrm{l}$ of $50 \mathrm{nM}$ AsCas12a, $62.5 \mathrm{nM}$ gRNA and $50 \mathrm{nM}$ custom ssDNA FQ substrate. Reaction was incubated at $37^{\circ} \mathrm{C}$ for $1 \mathrm{~h}$ and fluorescence was measured using Tecan Infinite Pro 200.

For investigating DETECTR's sensitivity, a titration experiment was performed. NCCR, sT and LT target region was amplified from plasmid RAZ2 (Addgene \#114382) and used as dsDNA target. This dsDNA was diluted to varying concentrations ranging from $10^{-8}$ to $10^{-15} \mathrm{M}$. Fluorophore quencher (FQ)-labeled reporter assay was performed as described previously using these targets with or without RPA. A variation of this mix, without any DNA target, but with gRNA and Cas12a enzyme and excess buffer was used as a "no target 

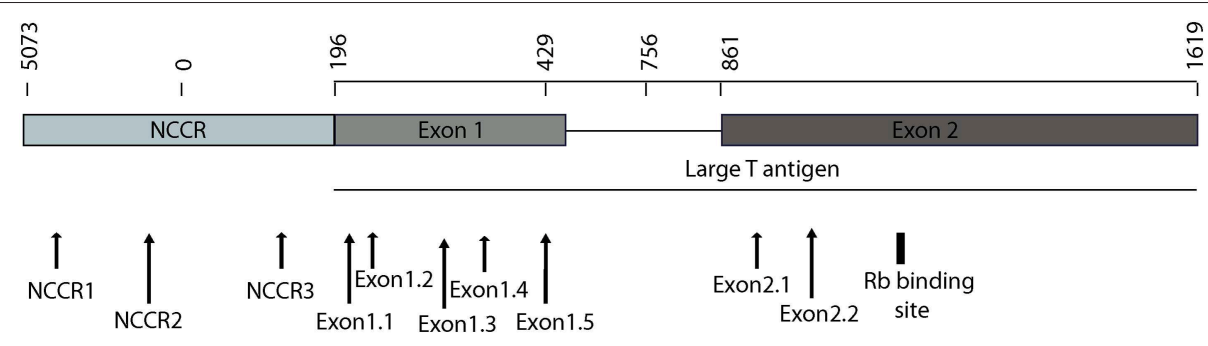

FIGURE 1 | MCV NCCR and early region map showing gRNA locations. The map above shows the location of 10 gRNAs (AsCas12a CRISPR based) that we designed and the specific sites on the MCV genome that they target.

TABLE 1 | AsCas12a_gRNAs against Merkel cell polyomavirus.

\begin{tabular}{|c|c|c|c|c|c|c|c|}
\hline & gRNA name & Strand & Sequence & PAM & MCV genome location* & Benchling score\# & RGEN score\# \\
\hline 1 & NCCR1 & Minus & AACAAGGGAGGCCCGGAGGC & ТाTC & $5156-5137$ & 98.01 & 66.3 \\
\hline 2 & NCCR2 & Minus & CTGGAGAGGCGGAGTTGAC & TाTC & $5239-5220$ & 98.24 & 74.2 \\
\hline 3 & NCCR3 & Minus & CAGAGGCCTCGGAGGCTAGG & TाTC & $26-7$ & 95.67 & 69.1 \\
\hline 4 & Exon1.1 & Minus & GGACTAAATCCATCTTGTCT & TाA & $208-189$ & 92.81 & 60.1 \\
\hline 5 & Exon1.2 & Plus & GAGATTGCTCCTAATTGTTA & ТाTA & $247-266$ & 93.40 & 62.4 \\
\hline 6 & Exon1.3 & Plus & GTCCTAAATAGGAAAGAAAGAGA & TाTA & $205-227$ & 79.10 & 73.9 \\
\hline 7 & Exon1.4 & Minus & CCCCTITATCAGGGTGATGCTIT & ТाТ & $334-312$ & 93.34 & 79.4 \\
\hline 8 & Exon1.5 & Minus & CTCCAAAGGGTGTTCAATTCCAT & TाTG & $374-352$ & 95.56 & 75.2 \\
\hline 9 & Exon2.1 & Plus & CCATCTAGGTTGACGAGGCC & तात & $853-872$ & 99.32 & 75.0 \\
\hline 10 & Exon2.2 & Minus & TGGATCTTGAGTTGGTCCCG & Тात & $962-981$ & 98.04 & 69.3 \\
\hline
\end{tabular}

*MCV Genome Location is based on MKL-1 genome (GenBank: FJ173815.1).

\#Benching and RGEN are softwares whose scores are out of 100.

control" for normalization of emitted fluorescence and fold change calculation.

\section{RESULTS}

\section{gRNAs Were Designed to Target All MCV Variants}

To detect the presence of integrated MCV DNA in MCC cells, we designed 10 guide RNAs compatible with AsCas12a. Three gRNAs are complementary to the Non-Coding Control Region (NCCR), 5 gRNAs target Exon 1 of $\mathrm{T}$ antigen and the remaining 2 gRNAs target Exon 2. We used MKL-1 MCV sequence (Genbank Accession \#: FJ173815.1), till the conserved Retinoblastoma (Rb) binding domain region to design these gRNAs (Figure 1 and Table 1). All gRNAs designed targeted conserved regions across MCV subtypes known so far (Schowalter et al., 2010; Matsushita et al., 2014a; Buck et al., 2016). Using Clustal Omega Multiple Sequence Alignment we checked $52 \mathrm{MCV}$ sequences (from NCBI) across all gRNA-targeting regions.

\section{Screening and Validation of gRNAs Targeting MCV Genome}

To access the cleavage efficiency of these gRNAs, we performed in vitro cleavage assays. We used the 2,193 bp long NCCR and T antigen region (derived from MS-1) as template/ DNA substrate for our assays. Six out of 10 gRNAs efficiently cleaved the target substrate as shown by the cleaved bands (Figure 2A).

Next, to validate the non-specific trans ssDNA shredding activity of AsCas12a, we used a non-complementary, circular, single stranded M13 DNA phage reporter. In the presence of MCV gRNA and complementary ssDNA target (cis-activator), AsCas12a led to indiscriminate shredding of single stranded M13 phage (Figure 2B). Thus, 5 MCV gRNAs: NCCR3, Exon 1.1, Exon 1.2, Exon 2.1, and Exon 2.2, were selected for further studies for detecting MCV.

\section{Detection of MCV via Fluorescence Measurement}

To develop a MCV DNA detection system, we used a fluorophore quencher (FQ)-labeled reporter assay. We assembled AsCas12a with its MCV gRNA and a complementary dsDNA cis target. We introduced a non-specific ssDNA FQ reporter to this reaction. In the presence of gRNA targeting MCV DNA and complementary target MCV DNA, AsCas12a cleaved the ssDNA FQ reporter as observed through the emitted fluorescence. We found that all the five selected gRNAs showed significant fold change in emitted fluorescence as compared to no gRNA reaction and HPV L1 targeting gRNA as controls (Figure 3 and Supplementary Figure 1). HPV L1 targeting gRNA and matching template were used as a positive control for the assays (Supplementary Figure 2; Chen et al., 2018). 
We proceeded further with 2 gRNAs: NCCR 3 and Exon 1.1 after validating them against "No Target control" (Supplementary Figure 3). The DETECTR from Prof. Doudna's
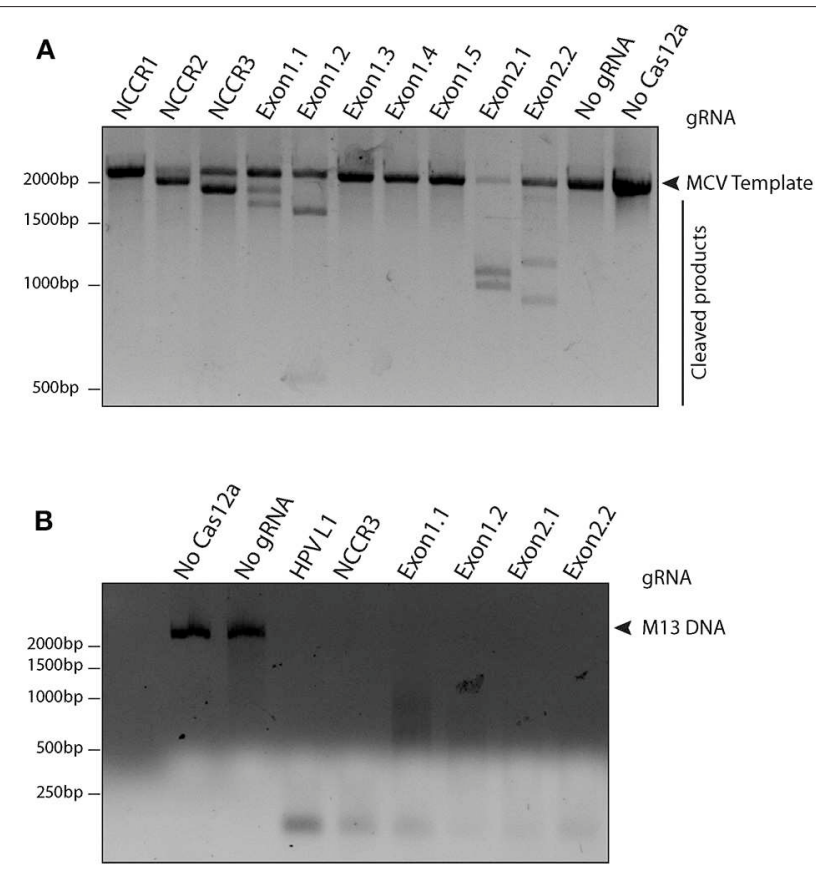

FIGURE 2 | Screening gRNAs for efficient cleavage. (A) in vitro cleavage (IVC) assay for all gRNAs. An MCV template of 2,193 bp spanning the NCCR region to the RB-binding site of $\mathrm{T}$ antigen was used for the assay (shown by the arrow). Of the $10 \mathrm{gRNAs}$ tested, six showed efficient cleavage that resulted in visible chopped bands on a $2 \%$ agarose gel. (B) Non-specific trans ssDNA shredding activity of AsCas12a-gRNA. M13 DNA phage reporter was subjected to MCV gRNA, complementary ssDNA target (cis-activator) and AsCas12a. All five gRNAs, namely NCCR3, Exon1.1, Exon1.2, Exon2.1, and Exon2.2 resulted in indiscriminate shredding of single-stranded M13 DNA. group, couples Recombinase Polymerase with Cas12a based DNA detection (Chen et al., 2018). To investigate the sensitivity of our MCV DETECTR system, we used a plasmid containing the MS-1 NCCR and T antigen region with varying concentrations (range- $10^{-15}-10^{-8} \mathrm{M}$ ). We performed Cas12a based detection using these targets with and without Recombinase Polymerase Amplification. Our results show that the MCV DETECTR (RPA + Cas12a) system we created can efficiently detect MCV DNA up to femtomolar concentration (Figure 4).

\section{DETECTR Can Diagnose MCV in MCC Genomic DNA}

Next, we investigated whether DETECTR can specifically and efficiently detect MCV in MCV positive MCC cell's genomic DNA. We extracted genomic DNA from various cultured human cells infected with MCV (MKL-1, MS-1, WaGa) or without MCV (MCC26, BJhTERT, U2OS). Whereas, MKL-1, MS-1, WaGa and MCC26 are all Merkel cell carcinoma cell lines, BJhTERT is an immortalized fibroblast cell line and U2OS is an osteosarcoma cell line.

We performed MCV detection in these samples using AsCas12a DETECTR (RPA + Cas12a). We found that although only Cas12a-gRNA complex was not sensitive enough to identify MCV, DETECTR unambiguously detected MCV in MKL-1, MS-1, and WaGa cells (Figure 5 and Supplementary Figure 4). Also, there was no significant signal in the presence of noncomplimentary DNA from MCV negative cells. Thus, we show that the MCV DETECTR can be potentially used as a specific and efficient platform for diagnosing MCV in MCC tumors (Figure 6).

\section{DISCUSSION}

Currently, the methods used for the experimental detection or diagnosis of MCV in tumors include immunohistochemistry,
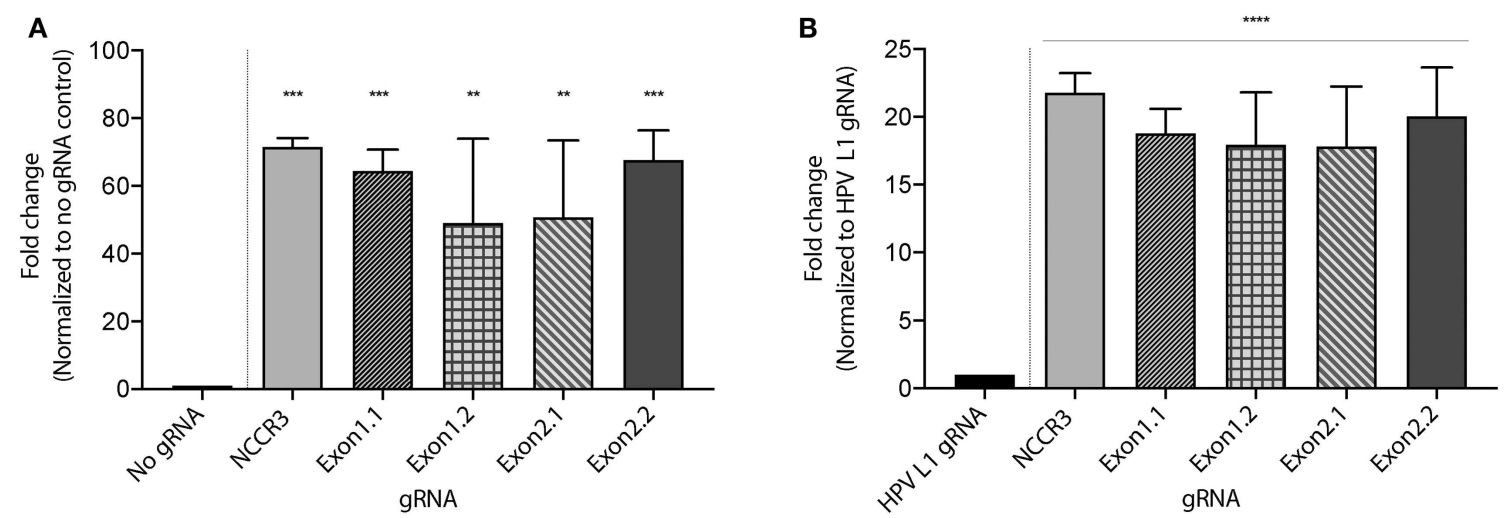

FIGURE 3 | MCV detection via fluorescence measurement. A Fluorophore-Quencher (FQ) labeled reporter assay was used to test the gRNA-AsCas12a combinations. MCV gRNA, AsCas12a and complementary dsDNA cis target were assembled and subjected to a custom ssDNA FQ reporter (excitation $485 \mathrm{~nm}$, emission $535 \mathrm{~nm}$ ). All five gRNAs showed significant fold change in emitted fluorescence (y axis) as compared to No gRNA control [ 48-fold and above, (A)] and HPV L1 gRNA control [ 18-fold and above, (B)]. Error bars represent SD for three independent experiments. One-way ANOVA with Dunnett test was performed for statistical analysis. (Adjusted $p$-values: $p_{\text {NCCR3 }}=0.0003, p_{\text {Exon1.1 }}=0.0007, p_{\text {Exon1 } .2}=0.0065, p_{\text {Exon2.1 }}=0.005$, and $p_{\text {Exon2.2 }}=0.0005,{ }^{\star \star} p<0.01,{ }^{\star \star *} p<0.001$ for $(\mathbf{A})$ and for $(\mathbf{B}){ }^{\star \star \star \star *} p$ $<0.0001)$. 

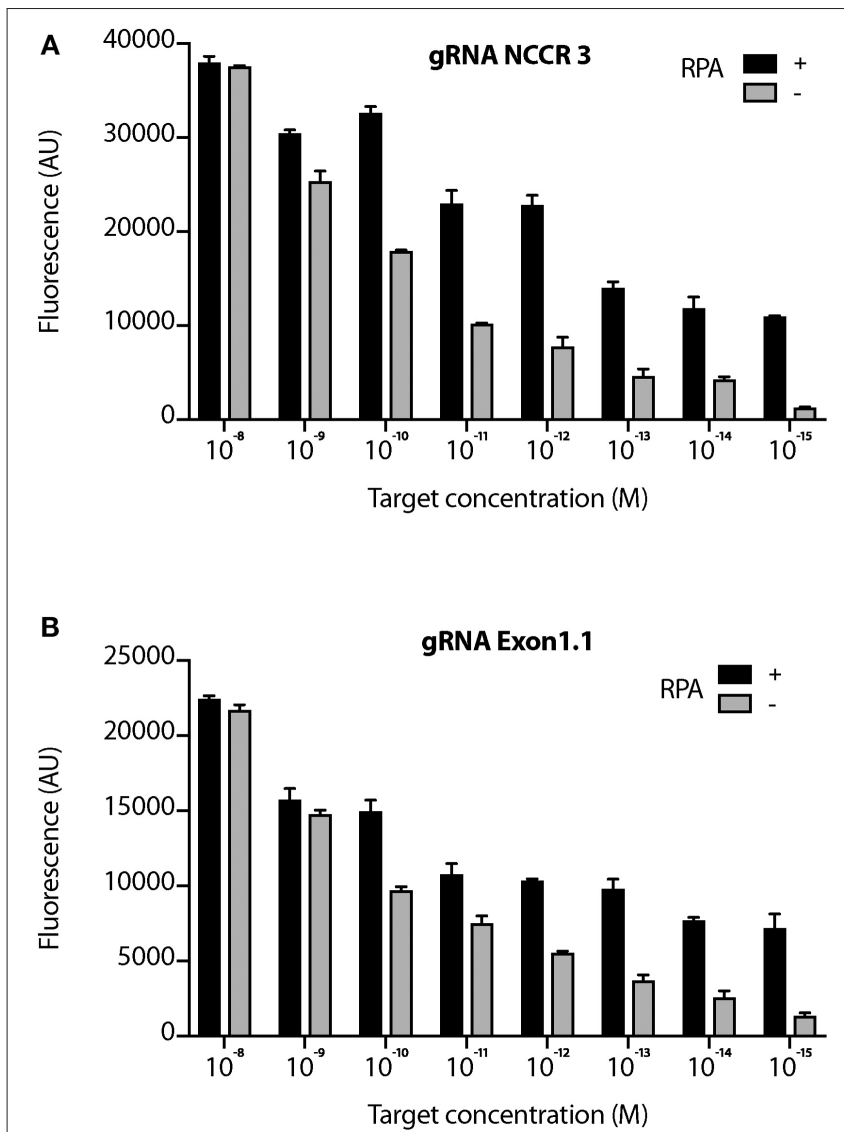

FIGURE 4 | Sensitivity of MCV gRNA to detect low copies of MCV. The Cas12a based detection of MCV was performed in the presence and absence of RPA (Recombinant Polymerase Amplification) with varying concentrations (range- $10^{-15}$ to $10^{-8} \mathrm{M}$ target concentration) for both (A) gRNA NCCR3 and (B) gRNA Exon1.1. y-axis represents background subtracted emitted fluorescence. Error bars represent SD for three independent experiments.

PCR, RNA, or DNA in situ hybridization and next-generation sequencing (NGS) (Shuda et al., 2009; Duncavage et al., 2011; Haugg et al., 2011; Rodig et al., 2012; Matsushita et al., 2014b; Wang et al., 2017; Harms et al., 2018). All of these assays vary considerably in terms of sensitivity and specificity for MCV detection in tumors. Immunohistochemical measurement of $\mathrm{T}$ antigen protein expression is the commonest approach for $\mathrm{MCV}$ detection and the antibody used for the same is usually CM2B4 (88\% sensitivity, 94\% specificity; Moshiri et al., 2017). Recently Moshiri et al. introduced a more efficient multimodal approach combining PCR and immunohistochemistry for detection of viral $\mathrm{T}$ antigens (Moshiri et al., 2017).

Although sensitive, these methods do have their drawbacks. For examples, CM2B4 often gives non-specific staining in both tonsillar and lymphoid tissues (Leroux-Kozal et al., 2015; Moshiri et al., 2017). Other antibodies that detect MCV T antigens, such as $\mathrm{Ab} 3$ and $\mathrm{Ab} 5$ have been reported, however none have been used as frequently as CM2B4 for detecting viral proteins in the clinic (Cheng et al., 2013; Harms et al., 2018; Arora et al., 2019).

PCR is another easy and common method used for $\mathrm{MCV}$ detection by many laboratories. In comparison with

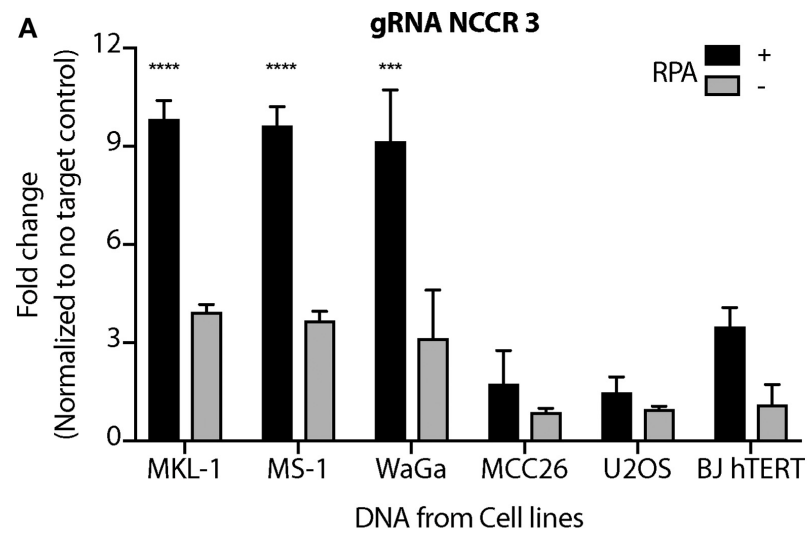

B

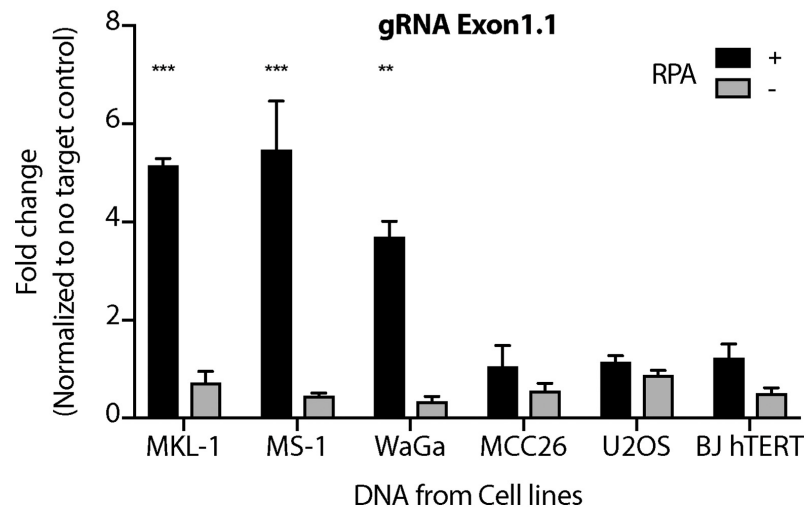

FIGURE 5 | MCV DETECTR in action. Genomic DNA from MCV positive MCC cell lines MKL-1, MS-1, WaGa; MCV negative MCC cell lines MCC26;

Osteosarcoma cell lines U2OS and immortalized Fibroblasts BJhTERT were extracted and subjected to the MCV DETECTR assay. With the use of RPA, MCV was detected significantly in all MCV MCC positive cell lines for both (A) gRNA NCCR and (B) gRNA Exon 1.1. Fluorescence was normalized to no-target control and fold change of emitted fluorescence plotted. Error bars represent SD for two independent experiments One-way ANOVA with Dunnett test was performed for statistical analysis $\left({ }^{\star \star} p<0.01,{ }^{\star \star \star} p<0.001,{ }^{\star \star \star \star} p<\right.$ 0.0001).

the multimodal approach, PCR-based amplification within the second exon of LT had $83 \%$ sensitivity and $81 \%$ specificity (Moshiri et al., 2017). Quantitative PCR (qPCR) extends this analysis and allows for estimation of number of integrated MCPyV copies per host cell genome (Katano et al., 2009; Sihto et al., 2009). MCPyV copy number estimates have been reported to range from $<1$ copy per 100 cells to thousands of copies per cell (Shuda et al., 2009; Rodig et al., 2012).

Technical factors, including inefficient PCR amplification owing to mutations in the integrated MCPyV genome, primer incompatibilities, low purity of tumor samples or the detection of infectious wild-type $\mathrm{MCPyV}$ in the adjacent non-malignant skin often confound the results for such tests (Eid et al., 2017; Wang et al., 2017). Besides, qPCR does not allow for visual confirmation that positive results are associated with tumor cells; hence, background MCV presence cannot be excluded in MCC tumors with low signal (Wang et al., 2017). 


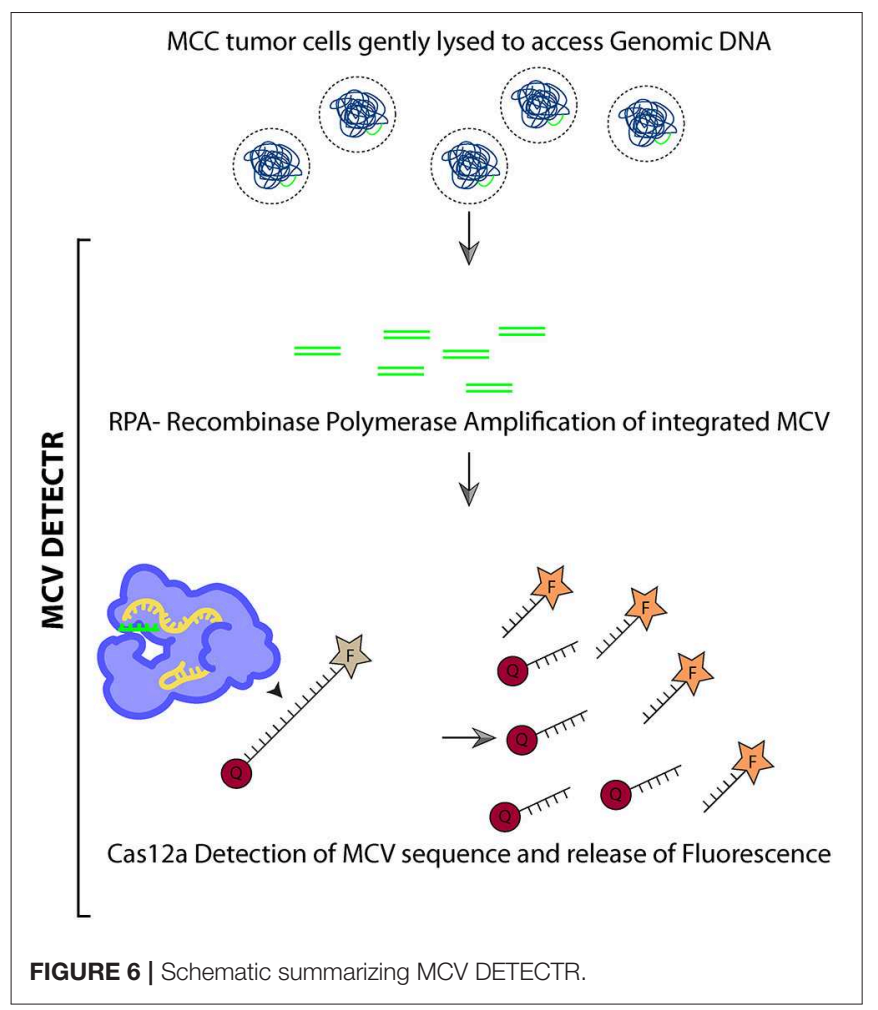

RNA in situ hybridization and NGS are other newer approaches being investigated for $\mathrm{MCPyV}$ detection. RNA in situ hybridization might provide PCR level sensitivity in conjunction with visual correlation with tissue morphology and the exclusion of background infection (Wang et al., 2017). NGS, on the other hand is effective in detecting MCPyV sequences, including tumor-specific truncating mutations and viral integration sites (Duncavage et al., 2011; Starrett et al., 2017). However, the time, expense and expertise required for these methods currently make these approaches impractical for many diagnostic and research laboratories.

The "cut and paste" function of CRISPR is popular and what the system is often associated with, after a guide RNA finds a target DNA sequence, the Cas nuclease makes a double-stranded cut and one can paste a sequence of choice at that site. The newer Cas 12 and Cas 13 have enabled researchers to use the "search" function of CRISPR in diagnostics (Liu et al., 2019). Platforms such as DETECTR by Jennifer Doudna's group (Chen et al., 2018) and SHERLOCK (Gootenberg et al., 2017, 2018) by Feng Zhang's group have leveraged this search functionality to get sensitive and specific diagnostic results for HPV and Dengue virus, respectively. Further studies to use CRISPR for Zika virus, Lassa fever, and even Ebola are underway (Chertow, 2018; Pan and Kraschel, 2018; Hadidi, 2019; Pickar-Oliver and Gersbach, 2019). The startups called Mammoth Biosciences and SHERLOCK Biosciences are such endeavors for providing better diagnostics for disease.

These non-editing applications of CRISPR have brought to light a precise, easy and efficient detection system for disease and here we have extended Dr. Doudna's group's studies to the tumor virus Merkel cell polyomavirus.

Our work offers a novel diagnostic method for MCV detection (Figure 6). Our MCV DETECTR approach is accurate, efficient, easy to use and rapid $(30 \mathrm{~min})$. It has a clear readout (fluorescence), specific and sensitive to femto-molar levels. The simplicity of this method, lack of complex sample preparation and no requirement for expensive equipment makes it superior to others currently being used. This CRISPR trans reporter system for DNA detection has opened a new applications door for CRISPR based technologies with rapid and specific detection of Merkel cell polyomavirus in patient samples. Additionally, combining this approach with immunohistochemistry and/or PCR will substantially improve the sensitivities of detection of MCV.

In our study, we tested the CRISPR system we developed for MCV on MCC cell lines for validation. Although robust and promising, further testing on clinical human MCC samples is needed and will add more value to the use of this system and further assist in taking it to clinical use as a diagnostic tool.

Thereby, our system is a simple platform for molecularly diagnosing whether a tumor is virus positive or negative. This information is not only an important causal indicator for Merkel tumors but also a powerful prognosis predictor. We hope that it will soon be incorporated into clinical practice and positively affect MCC studies.

\section{DATA AVAILABILITY STATEMENT}

All datasets generated for this study are included in the article/Supplementary Material.

\section{AUTHOR CONTRIBUTIONS}

RA and KG performed the experiments and wrote the manuscript. AV provided important reagents and performed the experiments. AV and SK provided intellectual support and valuable discussions. All authors listed have made a substantial, direct and intellectual contribution to the work, and approved it for publication.

\section{FUNDING}

This work was supported by the Wellcome Trust/DBT India Alliance (Early Career Award IA/E/14/1/501773 to RA).

\section{ACKNOWLEDGMENTS}

We are grateful to Dr. Debojyoti Chakraborty and Mohammed Azhar from IGIB, Delhi for guidance and advice on CRISPR related experiments. We thank Aurelie Jory for providing Cas12a protein for pilot experiments. Special thank you to Lamiya Dohadwala and Bhavana Nayer for technical assistance and to Prof. Sanjeev Galande for scientific comments. We also thank Dr. Anirudha Lakshminarasimhan, Divij Kinger, Dr. Alpana De, and Gayathri Rathinam from Tata Institute for Genetics and 
Society (TIGS), Bangalore for providing access and assistance in fluorescence measurements using Tecan machine. RA would also like to thank Zaina, Ziyah, and Karan for their love, strength, and support.

For Figure 6 the Innovative Genomics Institute (IGI) Glossary Icon Collection image for Cas12a was used. This is licensed under a Creative Commons Attribution- Non Commercial-ShareAlike 4.0 International Licence.

\section{REFERENCES}

Arora, R., Chang, Y., and Moore, P. S. (2012). MCV and Merkel cell carcinoma: a molecular success story. Curr. Opin. Virol. 2, 489-498. doi: 10.1016/j.coviro.2012.05.007

Arora, R., Rekhi, B., Chandrani, P., Krishna, S., and Dutt, A. (2019). Merkel cell polyomavirus is implicated in a subset of Merkel cell carcinomas, in the Indian subcontinent. Microb. Pathog. 137:103778. doi: 10.1016/j.micpath.2019.103778

Barrangou, R., Fremaux, C., Deveau, H., Richards, M., Boyaval, P., Moineau, S., et al. (2007). CRISPR provides acquired resistance against viruses in prokaryotes. Science 315, 1709-1712. doi: 10.1126/science.1138140

Becker, J. C., Stang, A., DeCaprio, J. A., Cerroni, L., Lebbe, C., Veness, M., et al. (2017). Merkel cell carcinoma. Nat. Rev. Dis. Prim. 3:17077. doi: $10.1038 /$ nrdp. 2017.77

Bhatia, K., Goedert, J. J., Modali, R., Preiss, L., and Ayers, L. W. (2010). Immunological detection of viral large $\mathrm{T}$ antigen identifies a subset of Merkel cell carcinoma tumors with higher viral abundance and better clinical outcome. Int. J. Cancer 127, 1493-1496. doi: 10.1002/ijc.25136

Brummer, G. C., Bowen, A. R., and Bowen, G. M. (2016). Merkel cell carcinoma: current issues regarding diagnosis, management, and emerging treatment strategies. Am. J. Clin. Dermatol. 17, 49-62. doi: 10.1007/s40257-015-0163-3

Buck, C. B., Van Doorslaer, K., Peretti, A., Geoghegan, E. M., Tisza, M. J., An, P., et al. (2016). The ancient evolutionary history of polyomaviruses. PLoS Pathog. 12:e1005574. doi: 10.1371/journal.ppat.1005574

Chen, J. S., Ma, E., Harrington, L. B., Da Costa, M., Tian, X., Palefsky, J. M., et al. (2018). CRISPR-Cas12a target binding unleashes indiscriminate single-stranded DNase activity. Science 360, 436-439. doi: 10.1126/science.aa r6245

Cheng, J., Rozenblatt-Rosen, O., Paulson, K. G., Nghiem, P., and DeCaprio, J. A. (2013). Merkel cell polyomavirus large $\mathrm{T}$ antigen has growth-promoting and inhibitory activities. J. Virol. 87, 6118-6126. doi: 10.1128/JVI.00385-13

Chertow, D. S. (2018). Next-generation diagnostics with CRISPR. Science 360, 381-382. doi: 10.1126/science.aat 4982

DeCaprio, J. A. (2017). Merkel cell polyomavirus and Merkel cell carcinoma. Philos. Trans. $R$ Soc. Lond. B Biol. Sci. 372:20160276. doi: 10.1098/rstb.2016.0276

Dong, D., Ren, K., Qiu, X., Zheng, J., Guo, M., Guan, X., et al. (2016). The crystal structure of Cpf1 in complex with CRISPR RNA. Nature 532, 522-526. doi: $10.1038 /$ nature 17944

Duncavage, E. J., Magrini, V., Becker, N., Armstrong, J. R., Demeter, R. T., Wylie, T., et al. (2011). Hybrid capture and next-generation sequencing identify viral integration sites from formalin-fixed, paraffinembedded tissue. J. Mol. Diagn. 13, 325-333. doi: 10.1016/j.jmoldx.2011. 01.006

Eid, M., Nguyen, J., and Brownell, I. (2017). Seeking standards for the detection of merkel cell polyomavirus and its clinical significance. J. Invest. Dermatol. 137, 797-799. doi: 10.1016/j.jid.2016.12.024

Engels, E. A., Frisch, M., Goedert, J. J., Biggar, R. J., and Miller, R. W. (2002). Merkel cell carcinoma and HIV infection. Lancet 359, 497-498. doi: 10.1016/S0140-6736(02)07668-7

Feng, H., Shuda, M., Chang, Y., and Moore, P. S. (2008). Clonal integration of a polyomavirus in human Merkel cell carcinoma. Science 319, 1096-1100. doi: $10.1126 /$ science. 1152586

Goh, G., Walradt, T., Markarov, V., Blom, A., Riaz, N., Doumani, R., et al. (2016). Mutational landscape of $\mathrm{MCPyV}$-positive and $\mathrm{MCPyV}$-negative Merkel cell
This manuscript has been released as a Pre-Print at bioRxiv (doi: https://doi.org/10.1101/770537).

\section{SUPPLEMENTARY MATERIAL}

The Supplementary Material for this article can be found online at: https://www.frontiersin.org/articles/10.3389/fmolb. 2020.00010/full\#supplementary-material

carcinomas with implications for immunotherapy. Oncotarget 7, 3403-3415. doi: 10.18632/oncotarget.6494

Gootenberg, J. S., Abudayyeh, O. O., Kellner, M. J., Joung, J., Collins, J. J., and Zhang, F. (2018). Multiplexed and portable nucleic acid detection platform with Cas13, Cas12a, and Csm6. Science 360, 439-444. doi: 10.1126/science.aaq0179

Gootenberg, J. S., Abudayyeh, O. O., Lee, J. W., Essletzbichler, P., Dy, A. J., Joung, J., et al. (2017). Nucleic acid detection with CRISPR-Cas13a/C2c2. Science 356, 438-442. doi: 10.1126/science.aam9321

Guastafierro, A., Feng, H., Thant, M., Kirkwood, J. M., Chang, Y., Moore, P. S., et al. (2013). Characterization of an early passage Merkel cell polyomaviruspositive Merkel cell carcinoma cell line, MS-1, and its growth in NOD scid gamma mice. J. Virol. Methods 187, 6-14. doi: 10.1016/j.jviromet.2012.10.001

Gupta, D., Bhattacharjee, O., Mandal, D., Sen, M. K., Dey, D., Dasgupta, A., et al. (2019). CRISPR-Cas9 system: a new-fangled dawn in gene editing. Life Sci. 232:116636. doi: 10.1016/j.lfs.2019.116636

Hadidi, A. (2019). Next-generation sequencing and CRISPR/Cas13 editing in viroid research and molecular diagnostics. Viruses 11:e120. doi: 10.3390/v11020120

Hall, B. J., Pincus, L. B., Yu, S. S., Oh, D. H., Wilson, A. R., and McCalmont, T. H. (2012). Immunohistochemical prognostication of Merkel cell carcinoma: p63 expression but not polyomavirus status correlates with outcome. J. Cutan. Pathol. 39, 911-917. doi: 10.1111/j.1600-0560.2012.01964.x

Harms, P. W., Collie, A. M., Hovelson, D. H., Cani, A. K., Verhaegen, M. E., Patel, R. M., et al. (2016). Next generation sequencing of Cytokeratin 20-negative Merkel cell carcinoma reveals ultraviolet-signature mutations and recurrent TP53 and RB1 inactivation. Mod. Pathol. 29, 240-248. doi: 10.1038/modpathol.2015.154

Harms, P. W., Harms, K. L., Moore, P. S., DeCaprio, J. A., Nghiem, P., Wong, M. K. K., et al. (2018). The biology and treatment of Merkel cell carcinoma: current understanding and research priorities. Nat. Rev. Clin. Oncol. 15, 763-776. doi: 10.1038/s41571-018-0103-2

Harms, P. W., Vats, P., Verhaegen, M. E., Robinson, D. R., Wu, Y. M., Dhanasekaran, S. M., et al. (2015). The distinctive mutational spectra of polyomavirus-negative Merkel cell carcinoma. Cancer Res. 75, 3720-3727. doi: 10.1158/0008-5472.CAN-15-0702

Haugg, A. M., Speel, E. J., Pantulu, N. D., Pallasch, C., Kurz, A. K., Kvasnicka, H. M., et al. (2011). Fluorescence in situ hybridization confirms the presence of Merkel cell polyomavirus in chronic lymphocytic leukemia cells. Blood 117, 5776-5777. doi: 10.1182/blood-2011-03-339895

Hodgson, N. C. (2005). Merkel cell carcinoma: changing incidence trends. J. Surg. Oncol. 89, 1-4. doi: 10.1002/jso.20167

Houben, R., Adam, C., Baeurle, A., Hesbacher, S., Grimm, J., Angermeyer, S., et al. (2012). An intact retinoblastoma protein-binding site in Merkel cell polyomavirus large $\mathrm{T}$ antigen is required for promoting growth of Merkel cell carcinoma cells. Int. J. Cancer 130, 847-856. doi: 10.1002/ijc.26076

Houben, R., Shuda, M., Weinkam, R., Schrama, D., Feng, H., Chang, Y., et al. (2010). Merkel cell polyomavirus-infected Merkel cell carcinoma cells require expression of viral $\mathrm{T}$ antigens. J. Virol. 84, 7064-7072. doi: 10.1128/JVI.02400-09

Hsu, P. D., Lander, E. S., and Zhang, F. (2014). Development and applications of CRISPR-Cas9 for genome engineering. Cell 157, 1262-1278. doi: 10.1016/j.cell.2014.05.010

Katano, H., Ito, H., Suzuki, Y., Nakamura, T., Sato, Y., Tsuji, T., et al. (2009). Detection of Merkel cell polyomavirus in Merkel cell carcinoma and Kaposi's sarcoma. J. Med. Virol. 81, 1951-1958. doi: 10.1002/jmv.21608 
Leroux-Kozal, V., Leveque, N., Brodard, V., Lesage, C., Dudez, O., Makeieff, M., et al. (2015). Merkel cell carcinoma: histopathologic and prognostic features according to the immunohistochemical expression of Merkel cell polyomavirus large T antigen correlated with viral load. Hum. Pathol. 46, 443-453. doi: 10.1016/j.humpath.2014.12.001

Liu, J. J., Orlova, N., Oakes, B. L., Ma, E., Spinner, H. B., Baney, K. L. M., et al. (2019). CasX enzymes comprise a distinct family of RNA-guided genome editors. Nature 566, 218-223. doi: 10.1038/s41586-019-0908-x

Matsushita, M., Iwasaki, T., Kuwamoto, S., Kato, M., Nagata, K., Murakami, I., et al. (2014a). Merkel cell polyomavirus (MCPyV) strains in Japanese merkel cell carcinomas (MCC) are distinct from Caucasian type MCPyVs: genetic variability and phylogeny of MCPyV genomes obtained from Japanese MCPyV-infected MCCs. Virus Genes 48, 233-242. doi: 10.1007/s11262013-1023-y

Matsushita, M., Nonaka, D., Iwasaki, T., Kuwamoto, S., Murakami, I., Kato, M., et al. (2014b). A new in situ hybridization and immunohistochemistry with a novel antibody to detect small T-antigen expressions of Merkel cell polyomavirus (MCPyV). Diagn. Pathol. 9:65. doi: 10.1186/17461596-9-65

Moore, P. S., and Chang, Y. (2017). Common commensal cancer viruses. PLoS Pathog. 13:e1006078. doi: 10.1371/journal.ppat.1006078

Moshiri, A. S., Doumani, R., Yelistratova, L., Blom, A., Lachance, K., Shinohara, M. M., et al. (2017). Polyomavirus-negative merkel cell carcinoma: a more aggressive subtype based on analysis of 282 cases using multimodal tumor virus detection. J. Invest. Dermatol. 137, 819-827. doi: 10.1016/j.jid.2016.10.028

Murugan, K., Babu, K., Sundaresan, R., Rajan, R., and Sashital, D. G. (2017). The revolution continues: newly discovered systems expand the CRISPR-Cas toolkit. Mol. Cell 68, 15-25. doi: 10.1016/j.molcel.2017.09.007

Pan, A., and Kraschel, K. L. (2018). CRISPR diagnostics: underappreciated uses in perinatology. Semin. Perinatol. 42, 525-530. doi: 10.1053/j.semperi.2018. 09.016

Pickar-Oliver, A., and Gersbach, C. A. (2019). The next generation of CRISPRCas technologies and applications. Nat. Rev. Mol. Cell Biol. 20, 490-507. doi: 10.1038/s41580-019-0131-5

Rodig, S. J., Cheng, J., Wardzala, J., DoRosario, A., Scanlon, J. J., Laga, A. C., et al. (2012). Improved detection suggests all Merkel cell carcinomas harbor Merkel polyomavirus. J. Clin. Invest. 122, 4645-4653. doi: 10.1172/JCI64116

Schowalter, R. M., Pastrana, D. V., Pumphrey, K. A., Moyer, A. L., and Buck, C. B. (2010). Merkel cell polyomavirus and two previously unknown polyomaviruses are chronically shed from human skin. Cell Host Microbe 7, 509-515. doi: $10.1016 /$ j.chom.2010.05.006
Schrama, D., Peitsch, W. K., Zapatka, M., Kneitz, H., Houben, R., Eib, S., et al. (2011). Merkel cell polyomavirus status is not associated with clinical course of Merkel cell carcinoma. J. Invest. Dermatol. 131, 1631-1638. doi: 10.1038/jid.2011.115

Shuda, M., Arora, R., Kwun, H. J., Feng, H., Sarid, R., Fernandez-Figueras, M. T. et al. (2009). Human Merkel cell polyomavirus infection I. MCV T antigen expression in Merkel cell carcinoma, lymphoid tissues and lymphoid tumors. Int. J. Cancer 125, 1243-1249. doi: 10.1002/ijc.24510

Shuda, M., Chang, Y., and Moore, P. S. (2014). Merkel cell polyomavirus-positive Merkel cell carcinoma requires viral small T-antigen for cell proliferation. J. Invest. Dermatol. 134, 1479-1481. doi: 10.1038/jid.2013.483

Shuda, M., Feng, H., Kwun, H. J., Rosen, S. T., Gjoerup, O., Moore, P. S., et al. (2008). T antigen mutations are a human tumor-specific signature for Merkel cell polyomavirus. Proc. Natl. Acad. Sci. U. S. A. 105, 16272-16277. doi: 10.1073/pnas.0806526105

Sihto, H., Kukko, H., Koljonen, V., Sankila, R., Bohling, T., and Joensuu, H. (2009). Clinical factors associated with Merkel cell polyomavirus infection in Merkel cell carcinoma. J. Natl. Cancer Inst. 101, 938-945. doi: 10.1093/jnci/djp139

Starrett, G. J., Marcelus, C., Cantalupo, P. G., Katz, J. P., Cheng, J., Akagi, K., et al. (2017). Merkel cell polyomavirus exhibits dominant control of the tumor genome and transcriptome in virus-associated Merkel cell carcinoma. MBio 8, e02079-16. doi: 10.1128/mBio.02079-16

Swarts, D. C., van der Oost, J., and Jinek, M. (2017). Structural basis for guide RNA processing and seed-dependent DNA targeting by CRISPR-Cas12a. Mol Cell 66, 221-233 e224. doi: 10.1016/j.molcel.2017.03.016

Wang, L., Harms, P. W., Palanisamy, N., Carskadon, S., Cao, X., Siddiqui, J., et al. (2017). Age and gender associations of virus positivity in Merkel cell carcinoma characterized using a novel RNA in situ hybridization assay. Clin. Cancer Res. 23, 5622-5630. doi: 10.1158/1078-0432.CCR-17-0299

Conflict of Interest: The authors declare that the research was conducted in the absence of any commercial or financial relationships that could be construed as a potential conflict of interest.

Copyright (c) 2020 Arora, Gupta, Vijaykumar and Krishna. This is an open-access article distributed under the terms of the Creative Commons Attribution License (CC $B Y)$. The use, distribution or reproduction in other forums is permitted, provided the original author(s) and the copyright owner(s) are credited and that the original publication in this journal is cited, in accordance with accepted academic practice. No use, distribution or reproduction is permitted which does not comply with these terms. 